\title{
Enhanced expression of glucose-regulated protein 78 correlates with malondialdehyde levels during the formation of liver cirrhosis in rats
}

\author{
YUN ZHANG ${ }^{1}$, HUIYING ZHANG ${ }^{2}$, ZHONGFU ZHAO ${ }^{1}$, MINLI LV ${ }^{3}$, JIANTAO JIA ${ }^{2}$, \\ LILI ZHANG ${ }^{2}$, XIAOXIA TIAN ${ }^{2}$, YUNXIA CHEN ${ }^{4}$, BAOHONG LI $^{2}$, \\ MINGSHE LIU ${ }^{1}$, DEWU HAN ${ }^{5}$ and CHENG JI ${ }^{6}$
}

\author{
${ }^{1}$ Institute of Hepatology; ${ }^{2}$ Department of Pathophysiology, Changzhi Medical College, Changzhi, Shanxi 046000; \\ ${ }^{3}$ ICU of The Second Hospital of Shanxi Medical University, Taiyuan, Shanxi 030001; ${ }^{4}$ Department of Microbiology, \\ Changzhi Medical College, Changzhi, Shanxi 046000; ${ }^{5}$ Institute of Hepatology, Shanxi Medical University, \\ Taiyuan, Shanxi 030001, P.R. China; ${ }^{6}$ USC Research Center for Liver Disease, Department of Medicine, \\ Keck School of Medicine, University of Southern California, Los Angeles, CA 90089, USA
}

Received January 23, 2015; Accepted August 7, 2015

DOI: $10.3892 /$ etm.2015.2783

\begin{abstract}
The aim of the present study was to explore the role of glucose-regulated protein 78 (GRP78) in the development of liver cirrhosis promoted by intestinal endotoxemia in rats. Fifty-one male Wistar rats were randomly divided into the liver cirrhosis 4-week, 6-week and 8-week groups and the normal control group at each time point. Liver cirrhosis was induced by employing multiple pathogenic factors in the rats. Blood and liver tissues were collected. The levels of alanine aminotransferase (ALT), homocysteine, endotoxin and tumor necrosis factor- $\alpha$ (TNF- $\alpha$ ) in the plasma, and TNF- $\alpha$, malondialdehyde (MDA) and procollagen type III peptide (PIIIP) in the liver tissues were determined. The mRNA and protein expression levels of GRP78 in the liver were detected using reverse transcription-quantitative polymerase chain reaction and immunohistochemistry. Morphological changes were observed through hematoxylin and eosin and van Gieson staining of the liver. Liver cirrhosis caused marked histopathological changes to the livers of the rats. Following significant increases in the levels of ALT, homocysteine, endotoxin and TNF- $\alpha$ in the plasma, and TNF- $\alpha$, MDA and PIIIP in the liver tissues of all experimental groups with the progression of liver cirrhosis, the mRNA and protein expression levels of GRP78 also gradually increased. In addition, correlation analysis indicated that the enhanced expression of GRP78 correlated with the MDA levels of the rats during the formation of liver cirrhosis.
\end{abstract}

Correspondence to: Professor Huiying Zhang, Department of Pathophysiology, Changzhi Medical College, 161 Jiefang East Street, Changzhi, Shanxi 046000, P.R. China

E-mail: zhanghy2001@163.com

Key words: liver cirrhosis, endotoxin, endoplasmic reticulum stress, homocysteine, glucose-regulated protein 78 , intestinal endotoxemia

\section{Introduction}

Glucose-regulated protein 78 (GRP78) is an important protein in the endoplasmic reticulum (ER). ER stress is a reaction that leads to cell death in organisms. During ER stress, GRP78 initiates a cellular self-defense mechanism, the unfolded protein response signaling cascade, which rebalances the functions of the ER, allowing the cells to survive under altered living conditions (1). Excessive and sustained ER stress triggers pathological processes, such as the nuclear factor- $\kappa \mathrm{B}$ $(\mathrm{NF}-\kappa \mathrm{B})$ signaling pathway, inflammatory response and programmed cell death, in which GRP78 acts as an important factor closely associated with the occurrence of multiple diseases $(2,3)$. Therefore, the role of GRP78 in liver diseases has been attracting increasing attention.

Hepatitis and liver cirrhosis are common diseases that are severely harmful to human health. During severe liver disease, the dysfunction or functional decline of the mechanical and immunological barriers of the intestinal mucosa causes a reduced defense capability against infections. This can easily result in infections of tissues and organs throughout the body, as well as intestinal endotoxemia (IETM) (4). IETM activates a variety of active substances secreted by Kupffer cells, such as cytokines, inflammatory mediators and free radicals; this leads to secondary liver tissue injury (5) and facilitates the formation of liver fibrosis or even liver cirrhosis. The main pathological feature of liver cirrhosis is the abnormal proliferation of collagen fibers, the synthesis of which is directly associated with the functions of the ER. Experimental studies have indicated that ER stress plays an important role in hepatitis, alcoholic liver injury and non-alcoholic fatty liver diseases (6-8).

In the present study, a rat model of liver cirrhosis was established through the inductive effects of composite pathogenic factors in order to investigate the effects that ER stress have on the pathogenesis of IETM-induced liver fibrosis and cirrhosis. 


\section{Materials and methods}

Reagents. $\mathrm{CCl}_{4}$ (analytically pure) was purchased from Fuyu Chemical Co., Ltd. (Tianjin, China), cholesterol from Tianjin Chemical Reagent Co.,Ltd.(Tianjin, China), and alanineaminotransferase (ALT) activity and malondialdehyde (MDA) assay kits were purchased from Nanjing Jiancheng Bioengineering Institute (Tianjin, China). Endotoxin Chromogenic End-point Tachypleus Amebocyte Lysate assay kit was obtained from Xiamen Horseshoe Crab Reagent Manufactory, Co., Ltd. (Xiamen, China), and the tumor necrosis factor- $\alpha$ (TNF- $\alpha$ ) radioimmunoassay kit from Beijing Purevalley Biotech Co., Ltd., (Beijing, China). The reverse transcription quantitative polymerase chain reaction (RT-qPCR) kit was purchased from Takara Biotechnology (Dalian) Co., Ltd. (Dalian, China) and the homocysteine (HCY) ELISA assay kit from AC Diagnostics, (San Diego, CA, USA). GRP78 rabbit anti-rat polyclonal antibody (G9043; Sigma-Aldrich, St. Louis, MO, USA) and immunohistochemistry streptavidin peroxidase (SP) kit were purchased from Beijing Biosynthesis Biotechnology Co., Ltd. (Beijing, China). Procollagen type III radioimmunoassay kit was purchased from Beijing Huaying Biotechnology Research Institute (Beijing, China). Commercial brand liquor, cornmeal and lard were all purchased from public markets.

Sample preparation. Fifty-one clean-grade healthy male Wistar rats, weighing 200-240 g, were provided by the Experimental Animal Center of Shanxi Medical University (Taiyuan, China). All animal experiments were conducted according to the ethical guidelines of Shanxi Medical University. The rats were randomly divided into four groups: The liver cirrhosis 4-week $(\mathrm{n}=11), 6$-week $(\mathrm{n}=11)$ and 8 -week $(n=11)$ groups, and the normal control group $(n=18)$, which received a normal diet. Rats in the normal control group were fed with standard feed and tap water. Rat liver cirrhosis models were constructed through the induction of composite pathogenic factors (9): i) The rats were fed with cornmeal blended with cholesterol ( $0.5 \%$ in weight), and in the first 2 weeks the feed was additionally blended with $20 \%$ lard; ii) the rats consumed only $5-15 \%$ alcohol as a beverage; and iii) the rats were injected subcutaneously on the back with $\mathrm{CCl}_{4}$ solution. On the first day of the experiment, the rats were injected with $\mathrm{CCl}_{4}$ at the amount of $0.5 \mathrm{ml} / 100 \mathrm{~g}$ body weight. They were then injected with $40 \% \mathrm{CCl}_{4}$ oil solution every 3 days at the amount of $0.3 \mathrm{ml} / 100 \mathrm{~g}$ body weight. Samples were collected at the end of weeks 4,6 and 8, respectively. Abdominal aortic blood was drawn in sterile and endotoxin-free conditions and was centrifuged at $1,200 \mathrm{xg}$ for $15 \mathrm{~min}$ at $4^{\circ} \mathrm{C}$ to separate the plasma, which was stored at $-70^{\circ} \mathrm{C}$. The rats were anesthetized with $3 \%$ pentobarbital sodium $(3 \mathrm{ml} / \mathrm{kg}$ body weight; Shanghai XiTang Biological Technology Co.,Ltd., Shanghai, China) and then sacrificed at 4-week, 6-week and 8-week after injection with $\mathrm{CCl} 4$, respectively. The rat livers were extracted and weighed and $10 \%$ of the liver tissue was cut and fixed with neutral formalin for histological examination. The rest of the liver tissue was immediately stored in liquid nitrogen.

Histopathology. Paraffin-embedded liver tissues were sectioned at $4 \mu \mathrm{m}$. Under an optical microscope (CX40;
Olympus Corporation, Tokyo, Japan), liver tissue injury was observed by examination of the slices stained with hematoxylin and eosin (H\&E) and the liver fibrosis status was visualized using van Gieson (VG) staining.

Determination of biochemical parameters in plasma and liver. For the plasma sample, ALT activity was determined by the Reitman and Frankel method (10). The levels of HCY were detected by ELISA assay, those of endotoxin using the Chromogenic End-point Tachypleus Amebocyte Lysate kit and those of TNF- $\alpha$ using radioimmunoassay. For the liver sample, tissue homogenates (10\%) were prepared for the examination of MDA, TNF- $\alpha$ and procollagen type III peptide (PIIIP) levels, according to the manufacturer's instructions of the RT-qPCR kit (Takara Biotechnology Co., Ltd.). Quantification of the proteins was performed using Coomassie brilliant blue staining.

$R T-q P C R$. Total RNA was extracted from the liver tissues of the rats by a one-step method using TRIzol ${ }^{\circledR}$ reagent (Gibco Life Technologies, Grand Island, NY, USA) and was then subjected to RT reaction using the RT-qPCR kit. The cDNA proliferation product of GAPDH was used as an internal control. The rat GAPDH primer sequences were as follows: Upstream, 5'-GGTCATCAACGGGAAACCC-3'; downstream, 5'-TCTGAGTGGCAGTGATGGCA-3'; the amplicon length was $450 \mathrm{bp}$. The rat GRP78 primer sequences were as follows: Upstream, 5'-GGAGGATGTGGGCACGGT GGTC-3'; downstream 5'-GTCATTCCAAGTGCGTCCGAT GAGG-3'. The amplicon length was $385 \mathrm{bp}$. The RT and qPCR reactions were conducted according to the manufacturer's instructions. PCR amplification conditions were as follows: Initial denaturation at $95^{\circ} \mathrm{C}$ for $60 \mathrm{sec}$, denaturation at $95^{\circ} \mathrm{C}$ for $15 \mathrm{sec}$ and annealing at $60^{\circ} \mathrm{C}$ for $60 \mathrm{sec}$, repeated for 40 cycles. The bands of the amplification products were scanned and analyzed using the Quantity One gel analysis system (Bio-Rad, Hercules, CA, USA). The absorbance ratio of GRP78 to GAPDH was calculated and served to indicate the relative content of the expressed GRP78 mRNA.

Immunohistochemistry. Paraffin-embedded slices were dewaxed and rehydrated through graded alcohols. The slices were then stained using SP according to the instructions provided with the immunohistochemistry kit. The primary GRP78 antibody (1:100) was replaced by phosphate-buffered saline for the negative control. GRP78 expression in the liver tissue was observed under a microscope, with the positive staining reaction observed as brown granules. Five slices were observed for each group of samples, and 10 fields of vision were recorded for each slice. Images were captured using a digital camera and analyzed using Image-Pro ${ }^{\circledR}$ plus 6.0 software (Media Cybernetics, Inc., Rockville, MD, USA) in order to determine the average optical density of the cells.

Statistical analysis. Data are presented as the mean \pm standard deviation. A one-way analysis of variance and LSD t-test were performed using SPSS 10.0 software (SPSS Inc., Chicago, IL, USA). Bivariate correlation method was selected and the Pearson correlation coefficient and significant correlation were calculated, with a significant correlation indicated by $<0.01$. In 

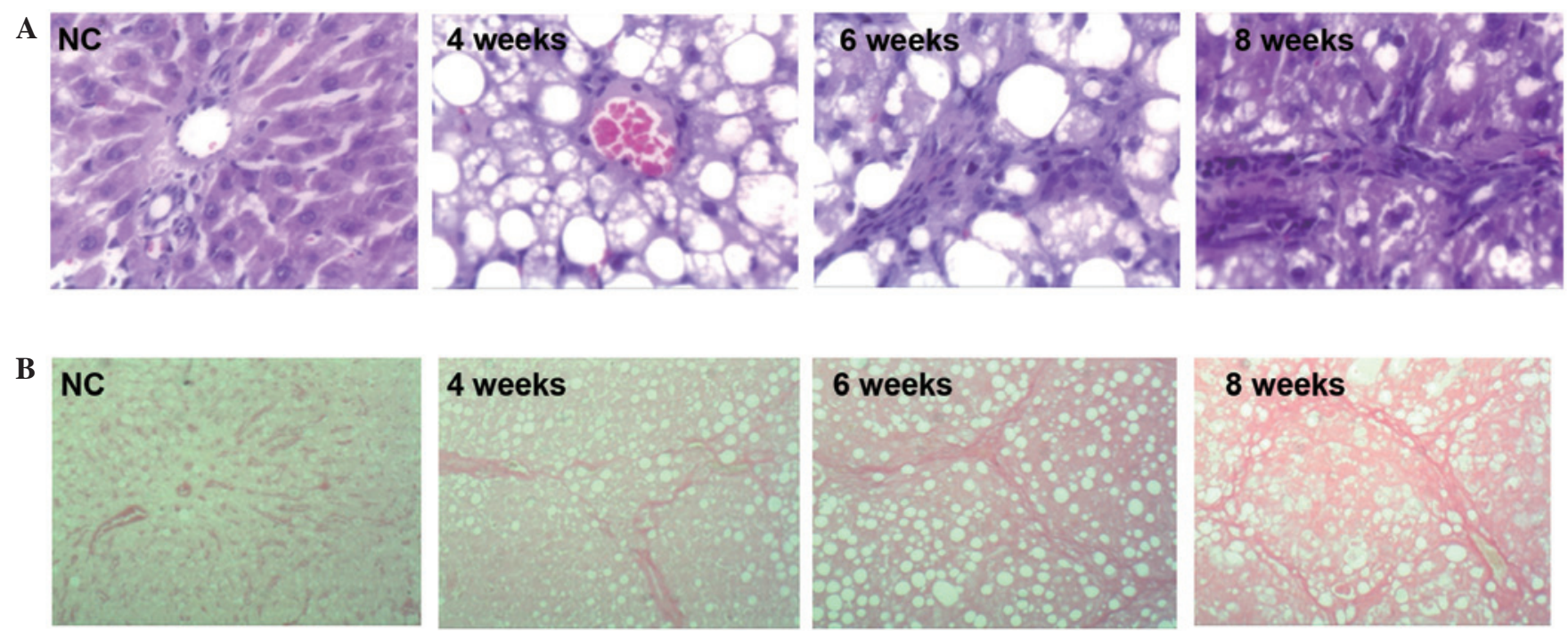

Figure 1. Histopathology of the rat livers. (A) Pathological changes of hepatocytes observed by hematoxylin and eosin staining (magnification, $\mathrm{x} 400$ ). (B) Changes in rat liver fibrosis observed by van Gieson staining (magnification, x100). The liver tissue fibrosis indices (collagen fiber area/total area) as determined by semi-quantitative analysis were as follows: NC, 3.10 \pm 1.32 ; 4 weeks, $6.52 \pm 1.96$; 6 weeks, $21.09 \pm 4.54 ; 8$ weeks, $30.30 \pm 2.2$ (all P<0.05 compared with the NC group). NC, normal control.

all other statistical analyses, $\mathrm{P}<0.05$ was considered to indicate a statistically significant difference.

\section{Results}

Liver cirrhosis induces changes in the general status and liver histopathology of rats. To investigate the general status of the rats, visual inspection was carried out. Rats of the normal control group appeared content and exhibited zero mortality, shiny hair, agile activities, quick responses, good appetite and normal urine and feces. Rats of the experimental groups exhibited messy, sparse, yellow and matted hair and hair loss, as well as listless behavior, decreased activity and appetite, limited feces, yellow urine, slow responses to external stimulations, and an infection rate of $18.2 \%(6 / 33)$, which was calculated based on the precess of abscesses. We observed the lungs and thoracic cavity of rats with liver cirrhosis at 4,6 and 8 weeks $(n=33)$. Anatomical observation indicated that 6 rats had pulmonary abscess, chest abscess or subdiaphragmatic abscess, and thus the infection rate was calculated to be $6 / 33$.

Histopathological investigation revealed that the livers of the rats in the normal control group were thin and sharp at the edge, ruddy in color, soft in texture and smooth on the surface, while the livers of the rats in the experimental groups had shrunk in size and were rounded at the edge, pale in color, hardened in texture and rough and uneven on the surface, which had numerous nodules.

H\&E staining revealed the following: In the control group the hepatic lobules were regular in structure, the arrangement of the hepatic cords was organized and they were distributed radially around the central vein, the cytoplasms of the liver cells were abundant and the nuclei were round and stained blue. No inflammatory cell infiltration was observed. In the liver cirrhosis 4 week group, the arrangement of the hepatic cell cords was disordered and fat vacuoles were observed. In the liver cirrhosis 6 week and 8 week groups, the structures of the hepatic lobules were no longer visible, irregular narrowing of the hepatic sinusoids was observed, the arrangement of the hepatic cells was disordered, steatosis was significant, bullous fat droplets were markedly accumulated and abundant fat vacuoles appeared in the cytoplasm. In addition, ballooning degeneration occurred in the cells surrounding the liver and inflammatory cell infiltration was observed, mainly in the portal area (Fig. 1A).

VG staining revealed the following: In the control group the hepatic lobules were regular in structure; the arrangement of the hepatic cords was organized, with only a few red filamentous fiber structures present in the central vein and portal area. In the liver cirrhosis 4 week group, a greater number of fibrous structures were observed, fibrous connective tissue proliferated and formed filamentous cords in the portal area and hepatic lobules were fragmented by sparse fiber bundles. In the liver cirrhosis 6 week group, more severe fibroplasia was observed and the width of the fibers varied. The fibers extended into the hepatic lobules in stellate shapes, dividing the liver into pseudolobules with various sizes and irregular shapes, and additional fibers appeared around the hepatic sinusoids, with those in the central vein and portal area being the most significant. In the liver cirrhosis 8 week group, fibrous tissues proliferated abundantly in the portal area. The majority of the fibrous tissues were connected to each other, with widened spaces between them. Furthermore, the normal structures of the liver tissue were altered (Fig. 1B).

Image analysis of the slices demonstrated that the content of collagen fibers in the liver tissues of the experimental groups was higher than that in the control group $(\mathrm{P}<0.05)$. These data suggest that liver cirrhosis caused significant histopathological changes to the livers of the rats.

Liver cirrhosis induces changes in the levels of ALT, HCY, endotoxin and TNF- $\alpha$ in rat plasma. During the formation of liver cirrhosis, the ALT activity levels of all three 
Table I. Levels of ALT, HCY, endotoxin and TNF- $\alpha$ in the plasma.

\begin{tabular}{llllll}
\hline Groups & $\mathrm{n}$ & \multicolumn{1}{c}{ ALT $(\mathrm{u} / \mathrm{l})$} & HCY $(\mu \mathrm{mol} / \mathrm{l})$ & Endotoxin $($ Eu/ml $)$ & TNF- $\alpha(\mathrm{ng} / \mathrm{ml})$ \\
\hline $\mathrm{NC}$ & 18 & $197.25 \pm 26.27$ & $26.23 \pm 4.10$ & $0.04 \pm 0.02$ & $1.35 \pm 0.40$ \\
4 week & 11 & $324.58 \pm 22.02^{\mathrm{a}}$ & $13.98 \pm 1.00^{\mathrm{a}}$ & $0.08 \pm 0.02$ & $2.13 \pm 0.18^{\mathrm{a}}$ \\
6 week & 11 & $380.43 \pm 30.90^{\mathrm{a}, \mathrm{b}}$ & $44.31 \pm 7.23^{\mathrm{a}}$ & $0.24 \pm 0.12^{\mathrm{a}, \mathrm{b}}$ & $2.10 \pm 0.23^{\mathrm{a}}$ \\
8 week & 11 & $364.24 \pm 29.89^{\mathrm{a}, \mathrm{b}}$ & $49.60 \pm 15.56^{\mathrm{a}, \mathrm{b}}$ & $0.26 \pm 0.18^{\mathrm{a}, \mathrm{b}}$ & $2.35 \pm 0.08^{\mathrm{a}-\mathrm{c}}$ \\
\hline
\end{tabular}

${ }^{a} \mathrm{P}<0.05$ compared with the NC group; ${ }^{b} \mathrm{P}<0.05$ compared with the 4 week group; ${ }^{\mathrm{c}} \mathrm{P}<0.05$ compared with the 6 week group. ALT, alanine aminotransferase; HCY, homocysteine; TNF- $\alpha$, tumor necrosis factor- $\alpha$; NC, normal control.

Table II. Levels of MDA, TNF- $\alpha$ and PIIIP in the liver tissues.

\begin{tabular}{lcccc}
\hline Groups & $\mathrm{n}$ & MDA $(\mathrm{nmol} / \mathrm{ml})$ & TNF- $\alpha(\mathrm{ng} / \mathrm{ml})$ & $\mathrm{PIIIP}(\mu \mathrm{g} / \mathrm{l})$ \\
\hline NC & 18 & $1.86 \pm 0.20$ & $0.92 \pm 0.23$ & $33.65 \pm 34.53$ \\
4 week & 11 & $4.20 \pm 0.53^{\mathrm{a}}$ & $1.21 \pm 0.05$ & $65.55 \pm 12.50^{\mathrm{a}}$ \\
6 week & 11 & $5.61 \pm 1.80^{\mathrm{a}, \mathrm{b}}$ & $1.74 \pm 0.77^{\mathrm{a}}$ & $78.74 \pm 28.60^{\mathrm{a}}$ \\
8 week & 11 & $11.06 \pm 1.99^{\mathrm{a}-\mathrm{c}}$ & $2.01 \pm 0.32^{\mathrm{a}, \mathrm{b}}$ & $75.54 \pm 29.11^{\mathrm{a}}$ \\
\hline
\end{tabular}

${ }^{\mathrm{a}} \mathrm{P}<0.05$ compared with the $\mathrm{NC}$ group; ${ }^{\mathrm{b}} \mathrm{P}<0.05$ compared with the 4 week group; ${ }^{\mathrm{C}} \mathrm{P}<0.05$ compared with the 6 week group. MDA, malondialdehyde; TNF- $\alpha$, tumor necrosis factor- $\alpha$; PIIIP, procollagen type III peptide; NC, normal control.
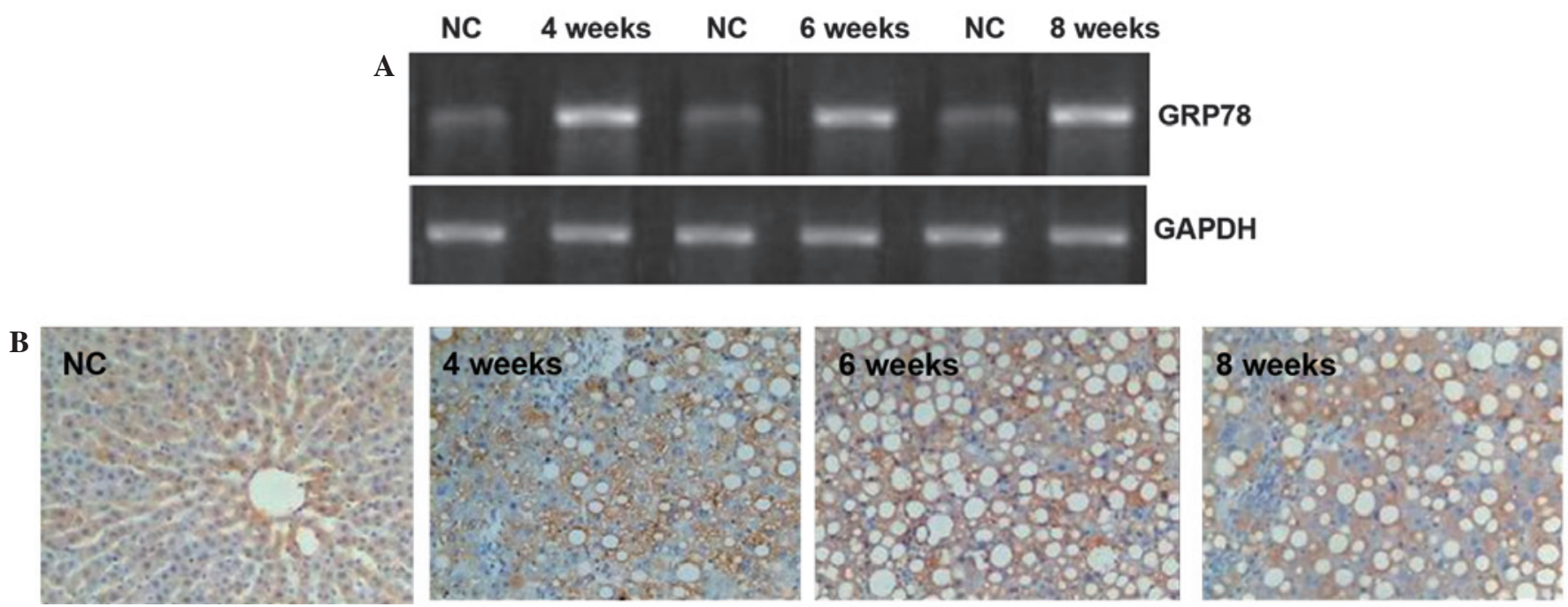

Figure 2. GRP78 expression in rat livers. (A) Gel assay for the detection of GRP78 mRNA in the rat liver tissues. Optical density values obtained using the Quantity One gel analysis system were as follows: NC, $1.16 \pm 0.03 ; 4$ weeks, $1.32 \pm 0.22 ; 6$ weeks, $1.36 \pm 0.08,8$ weeks, $1.47 \pm 0.12$ (all $\mathrm{P}<0.05$ compared with the NC group). (B) Immnunohistochemistry of GRP78 protein expression in the rat liver tissues (magnification, $x 400$ ). The number of GRP78 positive cells in the liver tissues of the rats as determined by semi-quantitative analysis were as follows: $\mathrm{NC}, 2.2 \pm 0.2 ; 4$ weeks, $8.2 \pm 1.5 ; 6$ weeks, 9.4 $\pm 1.0 ; 8$ weeks, 10.2 \pm 1.6 (all $\mathrm{P}<0.05$ compared with the NC group). GRP78, glucose-regulated protein 78; NC, normal control.

experimental groups were higher than that those in the control group $(\mathrm{P}<0.05)$. The levels of HCY, endotoxin and TNF- $\alpha$ in the plasma increased gradually, with those at the end of week 8 being significantly different from those in the control group $(\mathrm{P}<0.05$; Table I). These results suggest that liver cirrhosis increased the levels of ALT, HCY, endotoxin and TNF- $\alpha$ in the plasma of the rats.

Liver cirrhosis induces changes in the levels of MDA, TNF- $\alpha$ and PIIIP in the rat liver tissue homogenates. For the quantification of the MDA, TNF- $\alpha$ and PIIIP levels in liver tissue homogenates, the BCA protein assay kit was used to determine the protein concentration. As the liver cirrhosis progressed, the MDA levels were elevated; the MDA levels of all experimental groups were significantly different from those in the control group $(\mathrm{P}<0.05)$. The concentration of TNF- $\alpha$ was also markedly increased in the experimental groups, and the TNF- $\alpha$ concentrations of the liver cirrhosis 6 week and 8 week groups were significantly different from that of the control group $(\mathrm{P}<0.05)$. The PIIIP levels of all 
Table III. Correlation analysis.

\begin{tabular}{llcl}
\hline Factor 1 & \multicolumn{1}{c}{ Factor 2} & Correlation coefficient & P-value \\
\hline Endotoxin in plasma & MDA in liver homogenate & 0.861 & $<0.01$ \\
Endotoxin in plasma & HCY in the plasma & 0.895 & $<0.01$ \\
Endotoxin in plasma & GRP78 positive cell number & 0.833 & $<0.01$ \\
GRP78 protein & MDA in the plasma & 0.800 & $<0.01$ \\
GRP78 protein & HCY in the plasma & 0.617 & $<0.01$
\end{tabular}

MDA, malondialdehyde; HCY, homocysteine; PIIIP, procollagen type III peptide; GRP78, glucose-regulated protein 78.

experimental groups were significantly higher than those of the control group $(\mathrm{P}<0.05$; Table II). These data indicate that liver cirrhosis elevated the levels of MDA, TNF- $\alpha$ and PIIIP in the liver tissue.

Liver cirrhosis induces increases in the GRP78 mRNA and protein expression levels in rat liver tissues. In order to investigate the GRP78 mRNA expression in the liver tissue of the rats, RT-qPCR was performed (Fig. 2A). Positive bands of GRP78 mRNA were observed for all experimental groups and the control group. GRP78 mRNA transcription in the liver cirrhosis groups was gradually enhanced by the end of weeks 4, 6 and 8, respectively, and the increased levels were significantly different from that of the control group $(\mathrm{P}<0.05)$.

To visualize the GRP78 protein expression, immunohistochemistry was employed. In the control group, only a few GRP78-positive cells with brown granules were visible in the cytoplasm of cells in the liver tissues. By contrast, the number of GRP78-positive granules observed in the experimental groups was significantly increased compared with the number observed in the control group (Fig. 2B). Furthermore, fat vacuoles appeared in the cells, and positive staining was observed in the cytoplasm and cell membranes (Fig. 2B). These results suggest that the GRP78 protein expression in the liver tissue was increased by liver cirrhosis in the rats.

Correlation between increases in GRP78 expression and MDA levels during the formation of liver cirrhosis in rats. Statistical analysis was conducted to test the correlation between various factors during the formation of liver cirrhosis in the rats. Endotoxin levels in the plasma were found to correlate with MDA levels in the liver homogenates, HCY levels in the plasma and the GRP78 positive cell number. The GRP78 protein expression level was found to be positively correlated with the MDA and HCY levels in the plasma (Table III); therefore, the present data suggest that MDA levels in rats were affected by the elevation of the GRP78 protein expression during the formation of liver cirrhosis

\section{Discussion}

In the present study, the rat liver underwent pathological processes, including inflammatory cell infiltration, hepatic steatosis, fibrosis and even cirrhosis, following the impairment induced by composite pathogenic factors. The elevated levels of ALT indicated that the hepatocytes were severely injured, and the increased levels of plasma HCY implied that liver metabolism was dysfunctional. Yellow skin and mucosa and dark urine suggested that the secretory function of the liver was decreased. The elevated MDA levels in liver tissue homogenates demonstrated the occurrence of oxidative stress. Increased endotoxin levels in the plasma, combined with similar elevations of TNF- $\alpha$ in the plasma and liver tissue homogenates, demonstrated the formation of IETM. Liver injury induced by idiopathic pathogenic factors is known to lead to gastrointestinal disorders, intestinal bacterial overgrowth, the functional decline of mechanical and immunological barriers of the intestinal mucosa and injury of phagocytic function Kupffer cells, which subsequently act as important causes of IETM (11) and bacteremia.

It has previously been demonstrated that, following secondary liver injury, IETM formed during various liver diseases is the primary cause of the transformation of acute hepatitis to chronic and then severe hepatitis. Endotoxins not only are directly toxic to hepatocytes, but also cause further damage to the liver by sequentially binding with lipolysaccharide binding protein $\mathrm{CD} 14$ and the transmembrane signaling receptor toll-like receptor 4 , to activate endotoxin-related signaling pathways, such as the $\mathrm{NF}-\kappa \mathrm{B}$ pathway, and to facilitate the transcription, synthesis and release of various cytokines and chemical factors (12). Notably, TNF- $\alpha$ not only mediates multiple biological effects of endotoxin by itself, but also induces other inflammatory mediators to jointly take effect in inducing transforming growth factor- $\beta$, and promoting the activation and proliferation of hepatic stellate cells and their transformation to fibroblasts, thus increasing fibronectin and proteoglycan synthesis and facilitating liver fibrosis and cirrhosis (13). Liver fibrosis is the intermediate phase in the transformation from hepatitis and liver damage to liver cirrhosis and its pathology involves the excessive precipitation of extracellular matrix including collagen, glycoprotein and proteoglycan, among which collagen type I and III are the main components. It may, therefore, be hypothesized that IETM plays a key role in liver cirrhosis. For a long time, $\mathrm{NF}-\kappa \mathrm{B}$ was deemed to be a molecular target for antagonizing the biological effect of endotoxin (14); however, blocking the effect of endotoxin by targeting a single signaling molecule is unlikely to be successful due to the existence of a network among different signaling pathways; therefore, it is important for the mechanisms of cirrhosis caused by IETM to be elucidated, in order to provide effective strategies to prevent and treat liver cirrhosis. 
GRP78 is a molecule that appears in the earliest stage of the development of the endoplasmic reticulum (ER) and possesses important functions. Studies have discovered that GRP78 is a key factor in the ER that is closely associated with multiple diseases. GRP78 has been shown to be directly involved in the onset of hepatitis, alcoholic liver injury and non-alcoholic fatty liver disease (7-9). In the present study, it was found that in the formation of rat liver cirrhosis, GRP78 protein expression in the liver tissues increased with the progression of the disease, and the percentage increase correlated with the plasma levels of endotoxin, MDA and HCY. Studies have indicated that endotoxins cause ER stress directly or indirectly through the induction of oxidative stress, which could also be promoted by ER stress. These two types of stress interact with each other in the progression of the disease (15-17).

In the present study, IETM-induced oxidative stress and mitochondrial dysfunction may have led to an imbalance of $\mathrm{Ca}^{2+}$, while the endotoxin induced a great number of inflammatory mediators that are likely to cause the accumulation of unfolded proteins in the ER, or cause ER stress by insulin resistance or by increasing free fatty acids and hence, increased the expression of GRP78 (18). In previous studies of rats with acute and alcoholic liver injuries, increases in GRP78 protein expression coincided with hepatocyte apoptosis and pathological injuries $(19,20)$. Furthermore, the reduction of GRP78 protein expression induced by chlorogenic acid has been reported to significantly inhibit liver fibrosis (21). Prostaglindin E1 has also exhibited inhibitory effects on the precipitation of liver collagen, while effectively reducing GRP78 levels (22). In addition, hyper-homocysteinemia (HHCY), which is caused by methionine cycle disorders, is also an important cause of ER stress and the high expression of GRP78 (8). Ji et al (8) showed that $\mathrm{HCY}$ increased the production of intracellular $\mathrm{O}_{2}$ by promoting the release of inflammatory mediators, such as NF- $\kappa B$, interleukin- 6 and interleukin-1B, reduced nitric oxide levels and induced ER stress, thereby facilitating the occurrence of liver cirrhosis.

On the basis of the aforementioned findings, the present study suggests that intestinal endotoxin causes ER stress, directly or indirectly through oxidative stress, and sustained ER stress generates positive feedback to aggravate the injury and cause a continuous increase of GRP78 expression levels, which may trigger a sterol regulatory cascade reaction (18). This would lead to the abnormal accumulation of hepatic lipids, causing steatosis, inflammation, necrosis and apoptosis of the liver cells, being the key factor to promote liver fibrosis formation. In addition, HHCY, which occurred simultaneously, is also likely to play a crucial role in the formation of liver fibrosis.

A previous study has reported that the overexpression of GRP78 in the livers of obese rats, achieved using adenoviral vectors, decreased the cleavage of sterol regulatory element binding protein-1c, inhibited the expression of lipogenesis enzymes and significantly alleviated hepatic steatosis (23). This particular study further demonstrated the close association between GRP78 expression and liver fibrosis; however, it remains unclear whether GRP78 is a switch for fat metabolism promoting hepatic steatosis in the condition of pathological high expression, and inhibiting hepatic steatosis when its expression level exceeds the regulating levels in the organisms following human intervention.
In conclusion, the data of the present study indicate that IETM and HHCY aggravate liver injuries, possibly by triggering ER stress in liver tissues, and facilitate the formation of hepatic steatosis, fibrosis and even cirrhosis. Therefore, during the treatment of liver diseases, in addition to reducing the production of endotoxin and lowering plasma HCY levels, it is also important to regulate GRP78 protein expression levels, in order to rebalance ER functions and delay or stop the progression of liver fibrosis and cirrhosis.

\section{Acknowledgements}

This study was supported by grants from the National Natural Science Foundation of China (grant no. 81070339), the International Science and Technology Cooperation Project in Shanxi Province (grant no. 2010081068), the Director Funding of MOE Key Laboratory on Cellular Physiology Established by Province and Ministry in Shanxi Medical University (grant no. 2010-09) and the Returned Overseas Expert Foundation in Shanxi Province (grant no. 211-091). In addition, funding was obtained from the US National Institutes of Health (grant nos. R01AA018612 and R01AA014428).

\section{References}

1. Gonzalez-Gronow M, Selim MA, Papalas J and Pizzo SV: GRP78: A multifunctional receptor on the cell surface. Antioxid Redox Signal 11: 2299- 2306, 2009.

2. Görlach A, Klappa P and Kietzmann T: The endoplasmic reticulum: Folding,calcium homeostasis, signaling, and redox control. Antioxid Redox Signal 8: 1391-1418, 2006.

3. Ji C: Dissection of endoplasmic reticulum stress signaling in alcoholic and non-alcoholic liver injury. J Gastroenterol Hepatol 23 (Suppl 1): S16-S24, 2008.

4. Zhang HY, Han DW, Wang XG, Zhao YC, Zhou X and Zhao HZ: Experimental study on the role of endotoxin in the development of hepatopulmonary syndrome. World J Gastroenterol 11: 567-572, 2005.

5. Ito K, Kiyosawa N, Kumagai K, Manabe S, Matsunuma N and Yamoto T: Molecular mechanism investigation of cycloheximide-induced hepatocyte apoptosis in rat livers by morphological and microarray analysis. Toxicology 219: 175-186, 2006.

6. Esfandiari F, Villanueva JA, Wong DH, French SW and Halsted $\mathrm{CH}$ : Chronic ethanol feeding and folate deficiency activate hepatic endoplasmic reticulum stress pathway in micropigs. Am J Physiol Gastrointest Liver Physiol 289: G54-G63, 2005.

7. Gentile CL and Pagliassotti MJ: The role of fatty acids in the development and progression of Nonalcoholic fatty liver disease. J Nutr Biochem 19: 567-576, 2008.

8. Ji C and Kaplowitz N: Betaine decreases hyperhomocysteinemia, endoplasmic reticulum stress and liver injury in alcohol-fed mice. Gastroenterology 124: 1488-1499, 2003.

9. Zhang HY, Han DW, Zhao ZF, Liu MS, Wu YJ, Chen XM and Ji C: Multiple pathogenic factor-induced complications of cirrhosis in rats: A new model of hepatopulmonary syndrome with intestinal endotoxemia. World J Gastroenterol 13: 3500-3507, 2007.

10. Reitman S and Frankel S: A colorimetric method for the determination of serum glutamic oxaloacetic and glutamic pyruvic transaminase. Am J Clin Pathol 28: 56-63, 1957.

11. Zhang HY, Han de W, Su AR, Zhang LT, Zhao ZF, Ji JQ, Li BH and Ji C: Intestinal endotoxemia exerts a central role in development of hepatopulmonary syndrome in a cirrhotic rat model induced by multiple pathogenic factors. World J Gastroenterol 13: 6385-6395, 2007.

12. Xu CP, Liu J, Liu JC, Han DW, Zhang Y and Zhao YC: Dynamic changes and mechanism of intestinal endotoxemia in partially hepatectomized rats. World J Gastroenterol 13: 3592-3597, 2007.

13. Friedman SL: Mechanisms of hepatic fibrogenesis. Gastroenterology 134: 1655-1669, 2008. 
14. Kim JH, Kim YS, Hwang JW, Han YK, Lee JS, Kim SK, Jeon YJ, Moon SH, Jeon BT, Bahk YY and Park PJ: Sulfated chitosan oligosaccharides suppress LPS-induced NO production via JNK and NF-кB inactivation. Molecules 19: 18232-18247, 2014.

15. Chen J, Qin J, Liu X, Han Y, Yang Z, Chang X and Ji X: Nitric oxide-mediated neuronal apoptosis in rats with recurrent febrile seizures through endoplasmic reticulum stress pathway. Neurosci Lett 443: 134-139, 2008.

16. Zhang K: Integration of ER stress, oxidative stress and the inflammatory response in health and disease. Int J Clin Exp Med 3: 33-40, 2010

17. Ilieva EV, Naudí A, Kichev A, Ferrer I, Pamplona R and Portero-Otín M: Depletion of oxidative and endoplasmic reticulum stress regulators in Pick disease. Free Radic Biol Med 48: 1302-1310, 2010.

18. Hiramatsu N, Kasai A, Hayakawa K, Yao J, Kitamura M: Real-time detection and continuous monitoring of ER stress in vitro and in vivo by ES-TRAP: Evidence for systemic, transient ER stress during endotoxemia. Nucleic Acids Res 34: e93, 2006.
19. Wen T, Wu ZM, Liu Y, Tan YF, Ren F and Wu H: Upregulation of heme oxygenase-1 with hemin prevents D-galactosamine and lipopolysaccharide-induced acute hepatic injury in rats. Toxicology 237: 184-193, 2007.

20. Ji C and Kaplowitz N: Hyperhomocysteinemia, endoplasmic reticulum stress and alcoholic liver injury. World J Gastroenterol 10: 1699-1708, 2004.

21. Shi H, Dong L, Bai Y, Zhao J, Zhang Y and Zhang L: Chlorogenic acid against carbon tetrachloride-induced liver fibrosis in rats. Eur J Pharmacol 623: 119-124, 2009.

22. Li L and Zhu HG: Inhibition of prostaglandin E1 on acumulation of collagen type I and III in liver of rabit with schistosoma japonicum. Zhong Guo Pu Tong Wai Ke Za Zhi She 16: 577-580, 2007 (In Chinese).

23. Kammoun HL, Hainault I, Ferré P and Foufelle F: Nutritional related liver disease: Targeting the endoplasmic reticulum stress. Curr Opin Clin Nutr Metab Care 12: 575-582, 2009. 